\title{
Field performance of the malaria highly sensitive rapid diagnostic test in a setting of varying malaria transmission
}

\author{
Julia Mwesigwa ${ }^{1 *} \mathbb{D}$, Hannah Slater ${ }^{3,4}$, John Bradley ${ }^{5}$, Binta Saidy ${ }^{1}$, Fatima Ceesay ${ }^{1}$, Charles Whittaker ${ }^{3}$, \\ Ballah Kandeh ${ }^{6}$, Davis Nkwakamna' ${ }^{1}$ Chris Drakeley ${ }^{7}$, Jean-Pierre Van Geertruyden², Teun Bousema ${ }^{8}$, \\ Jane Achan ${ }^{1}$ and Umberto D'Alessandro ${ }^{1}$
}

\begin{abstract}
Background: The Gambia has successfully reduced malaria transmission. The human reservoir of infection could further decrease if malaria-infected individuals could be identified by highly sensitive, field-based, diagnostic tools and then treated.

Methods: A cross-sectional survey was done at the peak of the 2017 malaria season in 47 Gambian villages. From each village, 100 residents were randomly selected for finger-prick blood samples to detect Plasmodium falciparum infections using highly sensitive rapid diagnostic tests (HS-RDT) and PCR. The sensitivity and specificity of the HSRDT were estimated (assuming PCR as the gold standard) across varying transmission intensities and in different age groups. A deterministic, age-structured, dynamic model of malaria transmission was used to estimate the impact of mass testing and treatment (MTAT) with HS-RDT in four different scenarios of malaria prevalence by PCR: 5, 15, 30, and $60 \%$, and with seasonal transmission. The impact was compared both to MTAT with conventional RDT and mass drug administration (MDA).

Results: Malaria prevalence by HS-RDT was 15\% (570/3798; 95\% Cl 13.9-16.1). The HS-RDT sensitivity and specificity were 38.4\% (191/497, 95\% Cl 34.2-42.71) and 88.5\% (2922/3301; 95\% Cl 87.4-89.6), respectively. Sensitivity was the highest $(50.9 \%, 95 \% \mathrm{Cl} 43.3-58.5 \%$ ) in high prevalence villages (20-50\% by PCR). The model predicted that in very low transmission areas $(\leq 5 \%)$, three monthly rounds of MTAT with HS-RDT, starting towards the end of the dry season and testing 65 or $85 \%$ of the population for 2 consecutive years, would avert 62 or $78 \%$ of malaria cases (over 2 years), respectively. The effect of the intervention would be lower in a moderate transmission setting. In all settings, MDA would be superior to MTAT with HS-RDT which would be superior to MTAT with conventional RDT.

Conclusion: The HS-RDT's field sensitivity was modest and varied by transmission intensity. In low to very low transmission areas, three monthly rounds per year of MTAT with HS-RDT at 85\% coverage for 2 consecutive years would reduce malaria prevalence to such low levels that additional strategies may achieve elimination. The model prediction would need to be confirmed by cluster-randomized trials.
\end{abstract}

Keywords: Highly sensitive rapid diagnostic test, Malaria, Mass testing and treatment, Plasmodium falciparum, Transmission areas

\footnotetext{
*Correspondence: Julia.mwesigwa@lshtm.ac.uk

${ }^{1}$ Medical Research Council Unit The Gambia at London School

of Hygiene and Tropical Medicine, P.O. Box 273, Banjul, The Gambia

Full list of author information is available at the end of the article
} 


\section{Background}

After a decade of considerable success in malaria control, progress has stalled, with no further reduction of malaria cases between 2015 and 2017 [1]. The Gambia is one of the sub-Saharan African countries where the number of malaria cases has steadily declined due to the scale up of malaria control interventions [2, 3]. However, malaria transmission has not been interrupted, possibly because of the persistence of asymptomatic infections, some of them sub-microscopic (sub-patent), that occur in all endemic settings $[4,5]$. In The Gambia, $60 \%$ of infections identified during the dry season (January-June) and 30\% of those during the transmission season (July-December) were asymptomatic, with a third of these sub-patent [6]. As transmission declines, the proportion of sub-patent infections among infected and asymptomatic individuals may increase, although their contribution to transmission remains unclear $[4,7]$.

Strategies such as mass drug administration (MDA) or mass testing and treatment (MTAT) target the human reservoir of infection and may decrease malaria prevalence to such low levels that other strategies may achieve elimination. While MDA aims at administering a full anti-malarial treatment to the whole population, without screening for either symptoms or infections [8], MTAT would treat only positive individuals identified through mass testing, regardless of symptoms [9]. The low sensitivity of conventional rapid diagnostic tests (RDTs), its detection limit is approximately $100-200$ parasites/ $\mu \mathrm{l}$ and comparable to light microscopy [5, 10-13], remains a major challenge for the success of MTAT campaigns. In Burkina Faso, Ethiopia and Kenya, MTAT did not achieve significant or sustained reductions in malaria transmission in communities $[14,15]$ and school children $[16,17]$, partly because conventional RDTs would not detect lowdensity, sub-patent infections $[18,19]$.

Low-density infections can be identified by more sensitive molecular diagnostic methods that detect the parasites' nucleic acid. Depending on the blood volume examined and the molecular target, molecular tests can detect infections with parasite densities as low as 0.020.1 parasites $/ \mu \mathrm{l}[20]$. However, this approach is complex, expensive, and not easily field deployable as it requires skilled staff and a central laboratory $[18,21]$.

The highly sensitive RDTs (HS-RDT) were developed for the field detection of low-density infections, with a detection limit of $40-125 \mathrm{pg} / \mathrm{ml}$ histidine-rich protein-2 (HRP2), tenfold lower than that of conventional SD Bioline Malaria Ag P.f RDT (800-1000 pg/ml) [11]. When compared to quantitative reverse-transcription PCR (q) RT-PCR, HS-RDT's sensitivity was $44 \%$ in asymptomatic individuals in Myanmar (low transmission), 47\% in malaria naïve individuals infected experimentally with blood stage Plasmodium falciparum parasites, and 84\% in Ugandan asymptomatic children (high transmission); its specificity was greater than 96\% [22]. In Myanmar, under laboratory conditions, HS-RDT's sensitivity was $51 \%$ compared to the combined high-volume ultrasensitive qPCR and Quansys human malaria 4-plex enzymelinked immunosorbent assay (ELISA) tests, and 54\% when compared to only ultrasensitive qPCR. In the field, the HS-RDT sensitivity was $36 \%$ compared to ultra-sensitive qPCR; HS-RDTs had a twofold higher sensitivity than conventional RDTs and microscopy [18]. In Papua New Guinea, HS-RDT detected $51 \%$ of the $P$. falciparum infections detected by ultra-sensitive qPCR and $26 \%$ of $P$. falciparum infections detected by both standard qPCR and ultra-sensitive qPCR [19].

It is unclear what effect MTAT with HS-RDT would have on malaria transmission as a proportion of infected people would remain undetected and untreated. The objective of this study was to determine the field sensitivity and specificity of HS-RDT (reference test: var gene acidic terminal sequence (varATS) PCR. These values were used to predict the effect of MTAT campaigns with HS-RDT on malaria prevalence using a mathematical model.

\section{Methods}

\section{Study design and participants}

Malaria transmission in The Gambia is highly seasonal, with peak transmission from October to November. A cross-sectional survey was conducted over a month (7 November to 8 December 2017) in 47 villages in eastern Gambia. The selected villages with populations between 100 and 800 residents are included as part of the Health and Demographic Surveillance System (HDSS) (Fig. 1). In each village, individuals aged at least 6 months were randomly selected for the survey; in smaller villages, all the inhabitants were selected.

Individual finger-prick blood samples were collected for HS-RDT and diagnostic PCR, for the latter on filter paper to diagnose malaria infections. Selected participants underwent medical examination and axillary temperature was measured by a digital thermometer. Participants with a positive HS-RDT and fever (axillary temperature $\geq 37.5^{\circ} \mathrm{C}$ ) and/or history of fever in the previous $24 \mathrm{~h}$ were treated with artemether-lumefantrine, the first line treatment in The Gambia.

\section{Data management}

Data was collected and managed using the REDCap (Research Electronic Data Capture) data capture tool that has an intuitive interface for validated data entry, audit trails for tracking and exporting data. Individual data were completed for demographic characteristics, history 


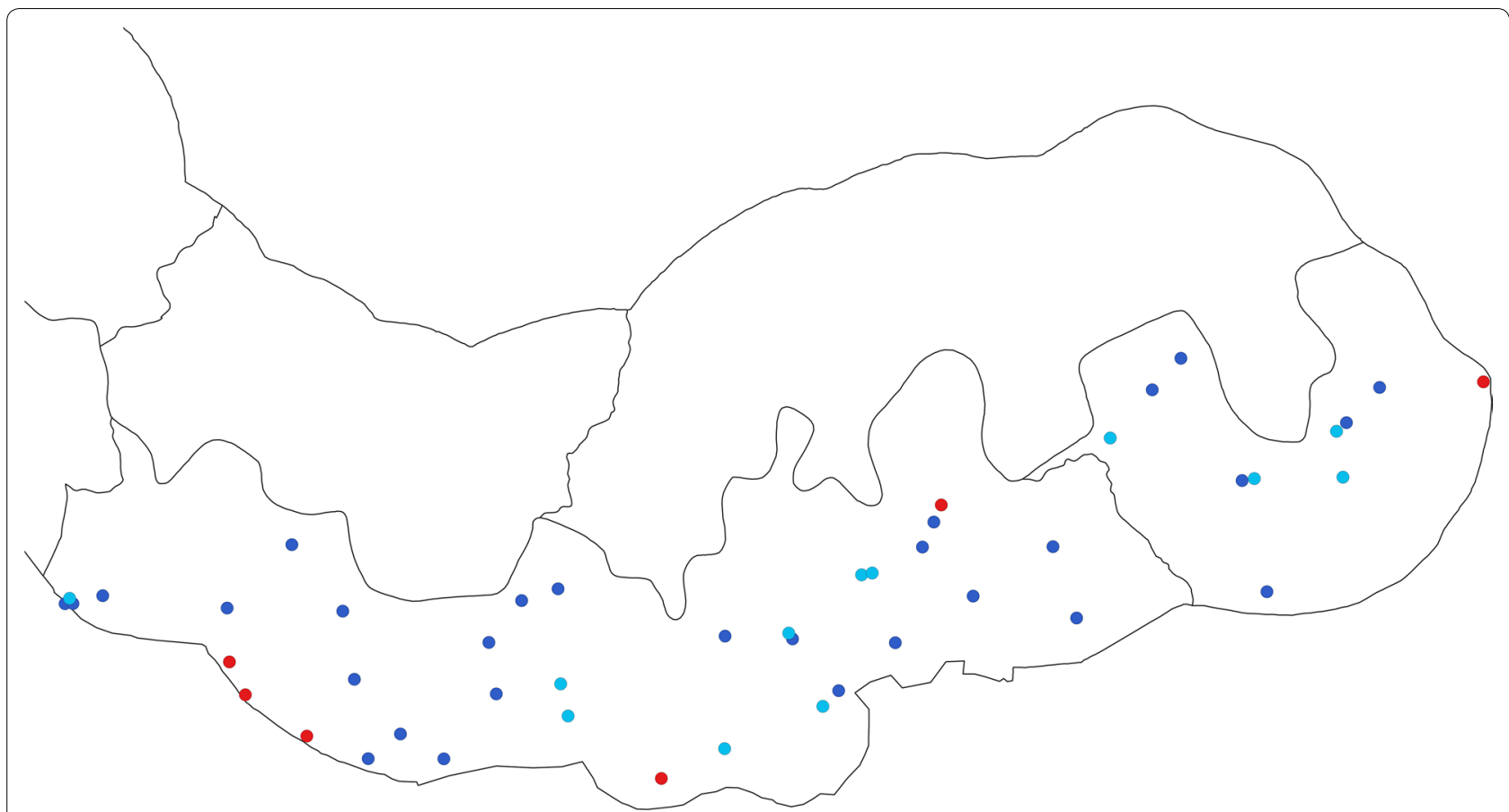

Fig. 1 Study sites in upper river region-south bank and Plasmodium falciparum infection prevalence by PCR. Filled light blue circle: very low prevalence $(<5 \%)$, filled dark blue circle: low-moderate transmission (5 to $<20 \%)$, filled red circle: high prevalence $(20-50 \%)$

of fever and recent anti-malarial use, axillary temperature, long-lasting insecticidal net (LLIN) ownership and use, and prior indoor residual spraying (IRS).

\section{Sample size}

Assuming malaria prevalence of $10 \%, 100$ individuals per village would be able to estimate malaria prevalence with a precision of $\pm 6 \%$.

\section{Malaria detection and laboratory analysis}

The HS-RDT was carried out following the manufacturer's recommendations. Briefly, a finger-prick blood sample was collected and delivered to the sample well in the test cassette. Four drops of buffer were added and the results were interpreted within 20 min by a study nurse [11].

The varATS assay was used to generate qualitative P. falciparum results. Briefly, three 3-mm dried blood spots were punched into 96-well plates and digested in $20 \mu \mathrm{l}$ of proteinase $\mathrm{K}$ and $180 \mu \mathrm{l}$ of ATL tissue lysis buffer solution. The Plasmodium DNA was extracted using the QIAamp 96 DNA QIAcube HT kit (Qiagen, Germany) and Qiacube $\mathrm{HT}^{\circledR}$ robot. Extracted DNA was eluted into $80 \mu \mathrm{l}$ of elution buffer and stored at $-20{ }^{\circ} \mathrm{C}$ till further use. For the analysis, the var gene acidic terminal sequence (varATS, 59 copies/genome) of P. falciparum was amplified [23]. All PCR reactions included
10 standards prepared from tenfold serially diluted samples containing known numbers of infected erythrocytes diluted in whole blood. The limit of detection of the PCR assay is approximately 0.2 parasites/ $\mu \mathrm{l}$ of blood [23]. The PCR output was analysed using the BioRad CFX Manager software.

\section{Statistical analysis}

Malaria prevalence was calculated by dividing the number of positive samples, either by HS-RDT or PCR, by the total number of tested samples. An asymptomatic infection was defined as an individual with positive HS-RDT or PCR without history of fever in the previous $24 \mathrm{~h}$ and axillary temperature $\leq 37.5{ }^{\circ} \mathrm{C}$ [24] at the time of blood collection. PCR was the reference test to estimate the diagnostic accuracy of HS-RDT. The sensitivity was estimated as follows: true positives/(true positives + false negatives); specificity as true negatives/(true negatives + false positives); positive predictive value as true positives/(true positive + false positives); negative predictive value as true negatives/(true negatives + false negatives). The Pearson's Chi squared test was used to compare the HS-RDT's sensitivity and specificity by age and transmission intensity. Univariate and multivariate logistic regression analysis was used to determine the odds of being "false negative" by age and the odds of being "false positive" by intensity of transmission. 
The regression models were adjusted for gender, IRS in the last 6 months and use of LLINs at night. Malaria prevalence by PCR was used as a proxy of transmission intensity, categorized as very low $(<5 \%$,) low-moderate (5 to $<20 \%)$ and high $(20-50 \%)$ transmission. The association between the HS-RDT sensitivity and transmission intensity was determined by fitting a line between the two quantities derived from a log-odds regression model [4] implemented within a Bayesian based framework. This framework was implemented in the statistical software package JAGS, and parameter inference based on Markov Chain Monte Carlo simulations using the $\mathrm{R}$ package rjags version 4-8 (https://CRAN.R-project.org/ package $=$ rjags). Infection status was defined by four categories: (1) malaria-infected individuals with detectable HRP2 levels: both PCR and HS-RDT positives; (2) individuals with a recently cleared infection, either naturally or by treatment, with residual HRP2: PCR negative and HS-RDT positive; (3) malaria-infected individuals without residual HPR2: PCR positive and HS-RDT negative test result; and, (4) non-infected individuals: both negative PCR and HS-RDT. The map of the study sites was generated using Quantum (Q)GIS version 7.5.0 with GRASS 7.4.4 programs. The administrative base map of The Gambia was created by downloading the "shapefiles" of The Gambia into QGIS and the administrative layers were generated. A final "shapefile" was created using the village coordinates and PCR infection prevalence and then layered onto the administrative map of The Gambia.

\section{Mathematical modelling}

An existing deterministic age-structured dynamic model of malaria transmission was used to estimate the impact of MTAT with HS-RDT (compared to no intervention) on malaria prevalence and the proportion of clinical malaria cases averted [25]. The model assumes newborns start as susceptible with some maternally acquired immunity, which decays within 6 months. After an infectious mosquito bite, individuals have a probability of developing symptomatic or asymptomatic malaria. The probability of becoming infected after an infectious bite depends on the individual's level of pre-erythrocytic immunity, and the probability of developing symptomatic or asymptomatic infection depends on their level of blood-stage immunity. The acquisition of both types of immunity depends on population-level of exposure. Individuals developing symptomatic malaria have a probability of being treated and then prophylactically protected. Untreated individuals with symptomatic infection are assumed to progress to asymptomatic infection after an average of 5 days. Asymptomatic individuals are assumed to remain $P$. falciparum infected for 310 days on average [26]. Infections are defined as either patent (detected by microscopy) or sub-patent (detected only by molecular method). Unless prophylactically protected by an antimalarial drug, individuals can be re-infected or superinfected at any time.

The model was extended to include MTAT with HSRDTs compared to either MTAT with conventional RDTs or MDA implemented as three monthly rounds per year for 2 consecutive years. The MTAT is implemented in the model whereby, at defined time points, a proportion of individuals (the intervention coverage) are tested. A proportion of infected individuals are assumed to test positive (determined by the diagnostic test's sensitivity), treated and moved to the 'prophylactic protection' compartment. It is assumed that there is non-random coverage of who partakes in each intervention round: the population is split in two groups, one which partakes in all three treatment rounds, and one which has random and uncorrelated coverage over the three rounds.

The HS-RDT's specificity is accounted for by assuming that a proportion of uninfected individuals will be HSRDT positive and will receive treatment and prophylactic protection. MDA is implemented in the model by assuming that at defined time points, a proportion (equal to the MDA coverage) of individuals are treated (regardless of infection status) and move to the 'prophylactic protection' compartment. Coverage between treatment rounds is assumed to be correlated as described above.

The model used the HS-RDT sensitivity and specificity values estimated by the survey. It was assumed that sensitivity of conventional RDT is $22 \%$ lower than the HSRDT and has 95\% specificity [22]. The model assumed recently treated individuals remain RDT or HS-RDT positive for 15 days [27] and 30 days [28], respectively. The probability of detection during this period is equal to the sensitivity of each test in asymptomatic individuals. In the simulations, dihydroartemisinin-piperaquine (DHA-PQ) was used for MTAT and MDA with the post-treatment prophylactic period assumed to be 30 days on average. Finally, seven scenarios are simulated: (i) no mass intervention; (ii) MTAT with conventional RDT at $65 \%$; (iii) $85 \%$ coverage; (iv) MTAT with HS-RDT at $65 \%$ coverage; (v) $85 \%$ coverage; (vi) MDA at $65 \%$ coverage, and (vii) $85 \%$ coverage. All interventions are implemented by three monthly round per year for 2 consecutive years, starting at the end of the dry season. All simulations are run according to different transmission intensity as determined by PCR-determined malaria prevalence namely; very low (5\%), low (15\%), moderate (30\%), and high (60\%).

\section{Consent}

Community verbal approval was followed by individual written consent for adults who also provided consent for 
children below 18 years of age. Children between 12 and 17 years provided assent. Ethical approval was obtained from the Gambia Government/Medical Research Council Joint Ethics Committee.

\section{Results}

A total of 4060 participants distributed in 47 villages were screened (Fig. 1); the median age was 13 years (IQR: $6,31) ; 55.7 \%(2258 / 4054)$ of study participants were female and coverage of control interventions (LLIN and IRS) was high (Table 1).

The prevalence of $P$. falciparum infection was $15.0 \%$ (570/3798; 95\% CI $13.9-16.1$ ) by HS-RDT and $13.1 \%$ (497/3798; 95\% CI $12.0-14.2)$ by PCR. HS-RDT's sensitivity and specificity were $38.4 \%$ (191/497; 95\% CI $34.2-42.7)$ and $88.5 \%$ (2922/3301; 95\% CI 87.4-89.6), respectively (Table 2 ).

\section{Table 1 Participants demographic characteristics}

\begin{tabular}{ll}
\hline Variable & N (\%) \\
\hline Age group (N=4024) & \\
0 to $<5$ & $667(16.6)$ \\
5 to $<10$ & $871(21.7)$ \\
10 to $<20$ & $996(24.8)$ \\
20 to $<40$ & $827(20.6)$ \\
40 to $<90$ & $663(16.5)$ \\
Gender (N=4054) & \\
Female & $2258(55.7)$ \\
Male & $1796(44.3)$ \\
Reported LLIN ownership $(\mathrm{N}=3998)$ & $3799(95.0)$ \\
Yes & $3512(87.6)$ \\
Used LLIN the previous night (N=4009) & \\
Yes & $3642(91.2)$ \\
Sprayed IRS in the previous 6 months (N=3992) & \\
Yes &
\end{tabular}

Among individuals with fever (temperature $\geq 37.5^{\circ} \mathrm{C}$ ) or history of fever, malaria prevalence by HS-RDT was $17.1 \%(200 / 1172 ; 95 \%$ CI $14.9-19.2)$ and $14.1 \%$ (369/2610; 95\% CI 12.8-15.5) in those without fever. HSRDT sensitivity was similar in individuals with $(41.5 \%$, 63/152; 95\% CI 33.6-49.3\%) or without (37.3\%, 128/343; 95\% CI 32.2-42.4) fever or history of fever in the previous $24 \mathrm{~h}(\mathrm{p}=0.38)$.

The HS-RDT sensitivity increased with increasing P. falciparum prevalence by PCR (Fig. 2), and was the highest, 50.9\% (85/167; 95\% CI 43.3-58.5) in high transmission villages (20-50\% prevalence) (Fig. 3). The HSRDT sensitivity was significantly lower and specificity higher in villages with low-moderate prevalence than in those with high prevalence $(\mathrm{p}<0.01)$ (Additional file 1 : Table S1).

The HS-RDT specificity was significantly lower in high $76.1 \%(229 / 301,95 \%$ CI 71.3-80.9) than in lowmoderate transmission $89.3 \%(1916 / 2146$, $95 \%$ CI $87.9-$ 90.6) $\left(\mathrm{X}^{2}=42.5, \mathrm{p}<0.01\right)$ or very low transmission $90.9 \%$ $\left(777 / 854,95 \%\right.$ CI 89.1-92.9) $\left(x^{2}=43.9, p<0.01\right)$ villages (Table 3). Additional file 1: Table S1 shows the comparison of the HS-RDT sensitivity and specificity by intensity of transmission.

More than half of PCR positive samples were negative by HS-RDT (306/497; 61.6\%, 95\% CI 57.3-65.8), with the largest proportion of 'false negatives' in villages with lowmoderate transmission. The odds of being false negative in the low-moderate and high prevalence villages was not different than in the very low prevalence villages. (Additional file 2: Table S2, Fig. 4).

The HS-RDT sensitivity did not differ between older children and adults but was significantly lower in children below 5 years than in older children and adults (Table 4, Fig. 5). The older children and adults had about $70 \%$ lower odds of being false negative, i.e., PCR positive and HS-RDT negative, than younger children (Table 5).

Table 2 Performance of highly sensitive rapid diagnostic tests as compared to PCR

\begin{tabular}{|c|c|c|c|}
\hline \multirow[t]{2}{*}{ PCR } & \multicolumn{2}{|l|}{ HS-RDT } & \multirow[t]{2}{*}{ Total } \\
\hline & HS-RDT positive & HS-RDT negative & \\
\hline Positive & 191 & 306 & 497 \\
\hline Negative & 379 & 2292 & 3301 \\
\hline \multirow[t]{2}{*}{ Total } & 570 & 3228 & 3798 \\
\hline & Value (\%) & $95 \% \mathrm{Cl}$ & \\
\hline Prevalence by HS-RDT & 15.0 & $13.9-16.1$ & \\
\hline Sensitivity & 38.4 & $34.2-42.7$ & \\
\hline Specificity & 88.5 & $87.4-89.6$ & \\
\hline Positive predictive value & 33.5 & $29.6-37.4$ & \\
\hline Negative predictive value & 90.5 & $89.5-91.5$ & \\
\hline
\end{tabular}




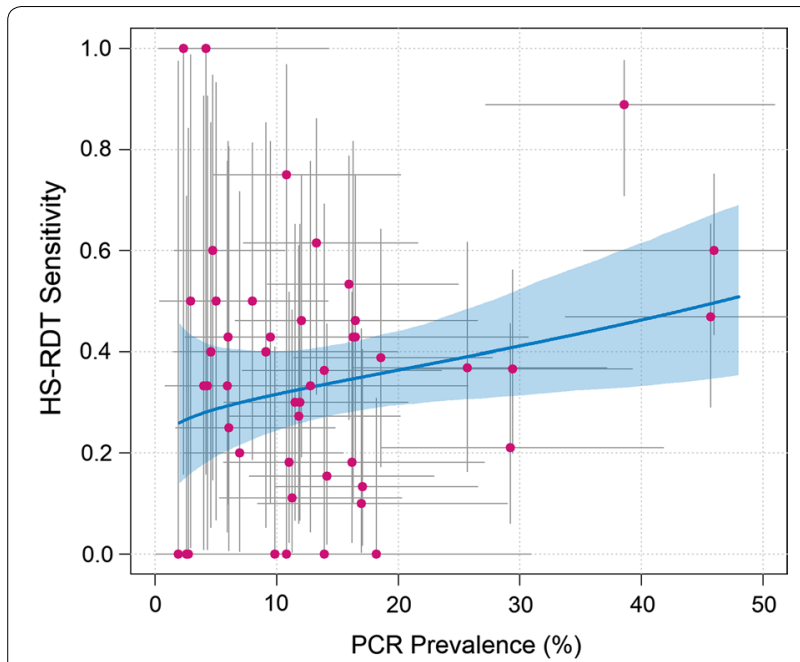

Fig. 2 Association between the highly sensitive rapid diagnostic test sensitivity and Plasmodium falciparum infection prevalence by PCR. Filled pink circle: each pink dot represents a village and the grey horizontal and vertical lines are $95 \%$ confidence intervals associated with the prevalence and sensitivity estimates, blue solid line: the fitted relationship between the two quantities derived from a log-odds regression model between PCR and HS-RDT prevalence implemented within a Bayesian framework. Full details of model structure have been previously published [25]

Overall, 11.5\% (379/3301) individuals were 'false positives', i.e. HS-RDT positive and PCR negative. This proportion increased with increasing malaria prevalence by
PCR with the odds of being false positive significantly higher in the high transmission villages than in very low transmission villages (Additional file 3: Table S3, Fig. 4). Only $3.7 \%$ (11/299; 95\% CI 1.6-5.8) false positive participants reported taking an anti-malarial treatment within the previous month, with similar proportions in lowmoderate $(4.8 \%, 8 / 78)$ and high $(4.2 \%, 3 / 72)$ transmission villages.

For the malaria transmission model, the HS-RDT sensitivity is assumed to be $38.4 \%$. The proportion of false positives (PCR negative and HS-RDT positive) was assumed to be $10 \%$. At very low prevalence $(\sim 5 \%)$, three monthly rounds of MTAT with HS-RDT per year at $85 \%$ coverage would decrease prevalence by $80 \%$, from 5 to around $1 \%$, up to 18 months after the final MTAT round (solid red line, Fig. 6a). In addition, $78.4 \%$ of clinical cases would be averted at the end of the 2 years. At $65 \%$ coverage malaria prevalence would decrease to $2 \%$ in 2 years averting $62.0 \%$ of malaria cases but it would return to pre-MTAT levels 12 months after the last monthly round of MTAT. The impact of MTAT with conventional RDT would be lower; at 85 and $65 \%$ coverage, it would avert 66.5 and $51.8 \%$ of cases, respectively. The effect of the intervention would be more marked for MDA as 93 and $80.5 \%$ of cases would be averted at 85 and $65 \%$ coverage, respectively (Additional file 4: Table S4 and Fig. 6a).

At low prevalence $(\sim 15 \%)$, three monthly rounds of MTAT with HS-RDT at $85 \%$ coverage would decrease prevalence to around 7 and $5 \%$ in the first and second

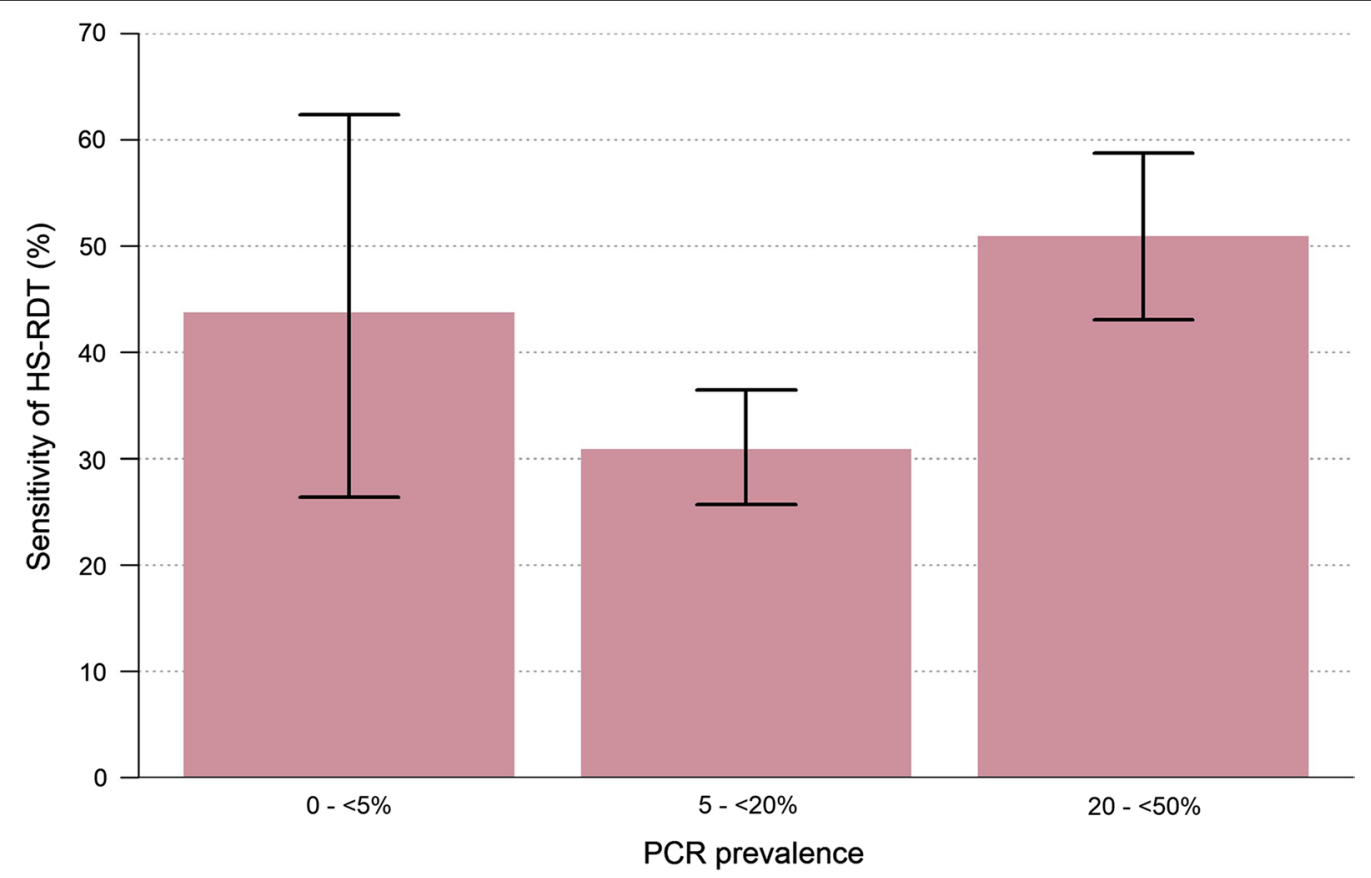

Fig. 3 Performance of highly sensitive rapid diagnostic test by transmission intensity 
Table 3 Performance of the highly sensitive rapid diagnostic test by malaria prevalence

\begin{tabular}{|c|c|c|c|c|c|c|c|c|c|}
\hline & \multirow{2}{*}{\multicolumn{3}{|c|}{$\begin{array}{l}\begin{array}{l}\text { Very low transmission } \\
\text { (prevalence }<5 \%)\end{array} \\
\text { HS-RDT }\end{array}$}} & \multirow{2}{*}{\multicolumn{3}{|c|}{$\begin{array}{l}\text { Low-moderate transmission } \\
\text { (prevalence } 5 \text { to }<20 \% \text { ) } \\
\text { HS-RDT }\end{array}$}} & \multirow{2}{*}{\multicolumn{3}{|c|}{$\begin{array}{l}\text { High transmission (prevalence } \\
20-50 \% \text { ) } \\
\text { HS-RDT }\end{array}$}} \\
\hline & & & & & & & & & \\
\hline & Positive & Negative & Total & Positive & Negative & Total & Positive & Negative & Total \\
\hline PCR positive & 14 & 18 & 32 & 92 & 206 & 298 & 85 & 82 & 167 \\
\hline PCR negative & 77 & 777 & 854 & 230 & 1916 & 2146 & 72 & 229 & 301 \\
\hline Total & 91 & 795 & 886 & 322 & 2122 & 2444 & 157 & 311 & 468 \\
\hline PCR prevalence & $3.6(2.5-5.1)$ & & & $12.2(10.9-13.5)$ & & & $35.7(31.3-40.2)$ & & \\
\hline Sensitivity $(95 \% \mathrm{Cl})$ & $43.8(26.4-62.3)$ & & & $30.9(25.6-36.1)$ & & & $50.9(43.3-58.5)$ & & \\
\hline Specificity $(95 \%$ CI) & $90.9(89.1-92.9)$ & & & $89.3(87.9-90.6)$ & & & $76.1(71.3-80.9)$ & & \\
\hline Positive predictive value $(95 \% \mathrm{Cl})$ & $15.4(8.7-24.5)$ & & & $28.6(23.6-33.5)$ & & & $54.1(46.4-61.9)$ & & \\
\hline Negative predictive value (95\% Cl) & $97.7(96.4-98.7)$ & & & $90.3(89.0-91.6)$ & & & $73.6(68.7-78.5)$ & & \\
\hline
\end{tabular}

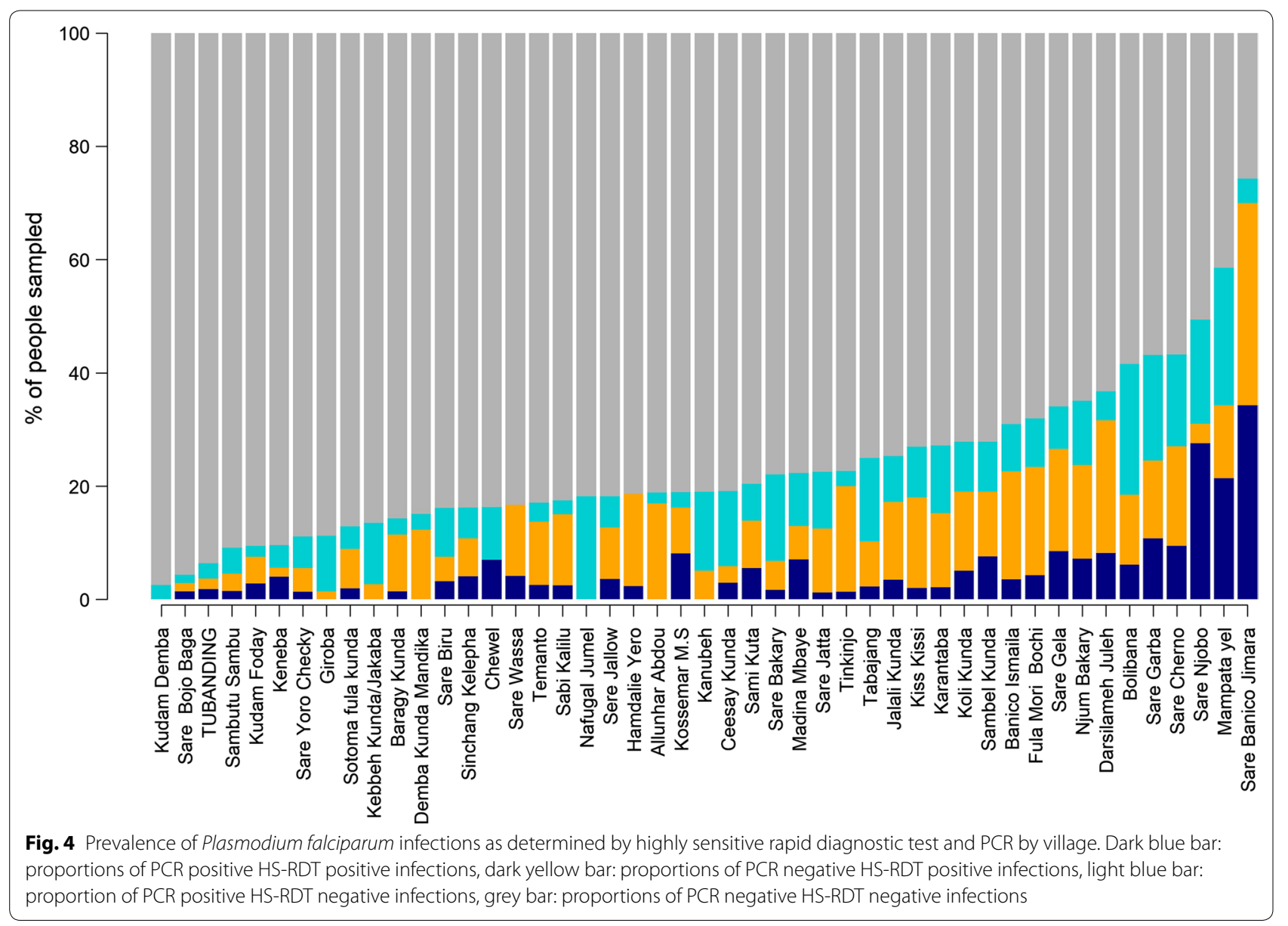

year, respectively. Prevalence would return to almost pre-MTAT levels 12 months after the last round if the intervention is not continued (solid red line, Fig. 6b); the intervention would avert $66.8 \%$ of malaria cases over 2 years (Additional file 4: Table S4). MDA at 85\% coverage would decrease malaria prevalence from 12 to about $5 \%$ in the first year and 3\% in the second year that would increase only after 18 months.

In moderate (prevalence $30 \%$ ) and high (prevalence $\sim 60 \%$ ) transmission areas, MTAT with HS-RDT 
Table 4 Performance of highly sensitive rapid diagnostic test by age

\begin{tabular}{|c|c|c|c|c|c|}
\hline & $\leq 5$ years & 5 to $<10$ years & 10 to $<18$ years & 18 to $<40$ years & $40-90$ years \\
\hline & $\begin{array}{l}\%, \mathrm{n} / \mathrm{N} \\
(95 \% \mathrm{Cl})\end{array}$ & $\begin{array}{l}\%, \mathrm{n} / \mathrm{N} \\
(95 \% \mathrm{Cl})\end{array}$ & $\begin{array}{l}\%, \mathrm{n} / \mathrm{N} \\
(95 \% \mathrm{Cl})\end{array}$ & $\begin{array}{l}\%, \mathrm{n} / \mathrm{N} \\
(95 \% \mathrm{Cl})\end{array}$ & $\begin{array}{l}\%, \mathrm{n} / \mathrm{N} \\
(95 \% \mathrm{Cl})\end{array}$ \\
\hline PCR prevalence & $\begin{array}{l}10.7 \%, 67 / 626 \\
(8.3-13.1)\end{array}$ & $\begin{array}{l}12.7 \%, 105 / 824 \\
(10.5-15.2)\end{array}$ & $\begin{array}{l}14.5 \%, 119 / 823 \\
(12.1-16.9)\end{array}$ & $\begin{array}{l}13.8 \%, 121 / 874 \\
(11.6-16.1)\end{array}$ & $\begin{array}{l}13.1,81 / 619 \\
(10.4-15.7)\end{array}$ \\
\hline Sensitivity & $\begin{array}{l}17.9 \%, 12 / 67 \\
(8.7-27.1)\end{array}$ & $\begin{array}{l}43.8 \%, 46 / 105 \\
(34.3-53.3)\end{array}$ & $\begin{array}{l}43.7 \%, 52 / 119 \\
(34.8-52.6)\end{array}$ & $\begin{array}{l}40.5 \%, 49 / 121 \\
(31.7-49.2)\end{array}$ & $\begin{array}{l}39.5 \%, 32 / 81 \\
(28.9-50.2)\end{array}$ \\
\hline Specificity & $\begin{array}{l}93.0 \%, 520 / 5599 \\
(90-95.1)\end{array}$ & $\begin{array}{l}87.1 \%, 626 / 719 \\
(84.6-89.5)\end{array}$ & $\begin{array}{l}84.5 \%, 597 / 704 \\
(81.8-87.6)\end{array}$ & $\begin{array}{l}87.9 \%, 662 / 753 \\
(85.6-90.3)\end{array}$ & $\begin{array}{l}91.8 \%, 494 / 538 \\
(89.5-94.1)\end{array}$ \\
\hline Positive predictive value & $\begin{array}{l}23.5 \%, 12 / 51 \\
(11.9-35.2)\end{array}$ & $\begin{array}{l}33.1 \%, 46 / 139 \\
(25.3-40.9)\end{array}$ & $\begin{array}{l}32.7 \%, 52 / 159 \\
(25.4-39.9)\end{array}$ & $\begin{array}{l}35.0 \%, 49 / 140 \\
(27.1-42.9)\end{array}$ & $\begin{array}{l}42.1 \%, 32 / 76 \\
(31.0-53.2)\end{array}$ \\
\hline Negative predictive value & $\begin{array}{l}90.4 \%, 520 / 575 \\
(88.03-92.8)\end{array}$ & $\begin{array}{l}91.4 \%, 626 / 685 \\
(89.3-93.4)\end{array}$ & $\begin{array}{l}89.9 \%, 597 / 664 \\
(87.6-92.2)\end{array}$ & $90.2 \%, 662 / 734$ (88.03-92.3) & $\begin{array}{l}90.9 \%, 494 / 543 \\
(88.6-93.4)\end{array}$ \\
\hline $\begin{array}{l}\text { Comparison of HS-RDT sensitivity of older } \\
\text { children and adults to children }<5 \text { years }\end{array}$ & 1 & $x^{2}=12.3, p<0.01$ & $x^{2}=12.6, p<0.01$ & $x^{2}=10, p<0.01$ & $x^{2}=8.2, p<0.01$ \\
\hline
\end{tabular}
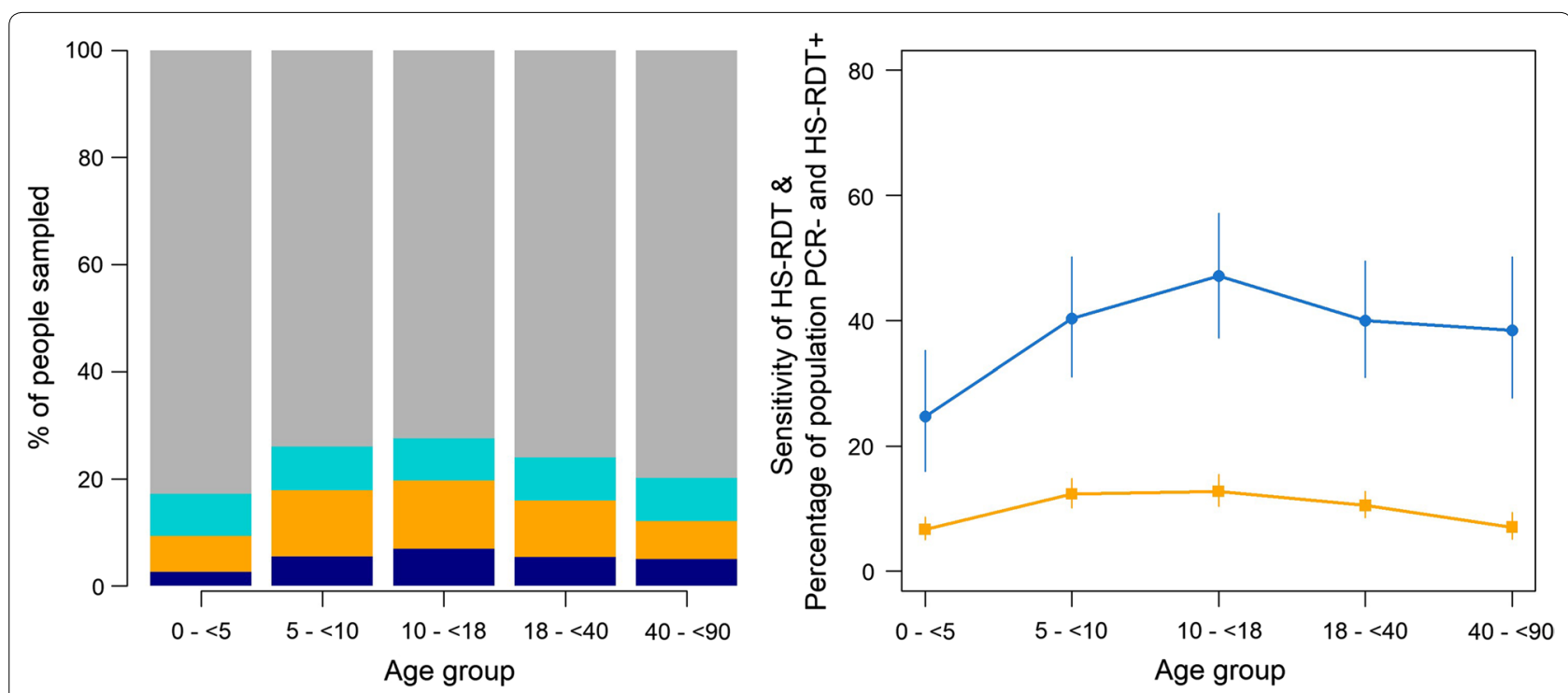

Fig. 5 Performance of highly sensitive rapid diagnostic test by age. a Dark blue bar: percentage of population that are PCR positive HS-RDT positive, dark yellow bar: percentage of population that are PCR negative HS-RDT positive, light blue bar: percentage of population that are PCR positive HS-RDT negative, grey bar: percentage of population that are PCR negative HS-RDT negative. $\mathbf{b}$ Solid blue line: sensitivity of HS-RDT (compared to PCR) for different age groups, with 95\% confidence intervals, solid red line: percentage of the population that are PCR negative and HS-RDT positive for different age groups, with 95\% confidence intervals

at $85 \%$ coverage would decrease malaria prevalence by about $60 \%$ during the intervention months; however, prevalence would rapidly return to pre-MTAT levels after the last round, i.e. 6 months in moderate and 3 months in high transmission areas (Fig. 6c, d). In moderate transmission settings, MTAT with a HS-RDT over 2 years would avert 53 and $40 \%$ of malaria cases at 85 and $65 \%$ coverage, respectively (Additional file 4: Table S4). The percentage of cases averted is lower for MTAT with a conventional RDT ( 42.8 and $33.4 \%$ at 85 and $65 \%$ coverage, respectively) and higher for MDA (80.6 and 61.9\% at 85 and $65 \%$ coverage, respectively). In all transmission settings, $85 \%$ coverage of MTAT with a HS-RDT had similar impact as MDA with a coverage of $65 \%$.

\section{Discussion}

HS-RDT sensitivity was low, missing $62 \%$ of infected individuals identified by PCR, but was similar to that reported from Myanmar (36.6\%) [18], Papua New Guinea (51\%) [19] and Ethiopia (33.9\%) [29], confirming the poor performance of this diagnostic test in detecting low-density malaria infections. Despite this, malaria prevalence 
Table 5 Risk of being false negative: PCR positive and HS-RDT negative by age, LLIN use and gender

\begin{tabular}{|c|c|c|c|c|c|}
\hline & $\begin{array}{l}\text { HS-RDT false negatives } \\
\mathrm{n} / \mathrm{N},(\%)\end{array}$ & OR $(95 \% \mathrm{Cl})$ & $p$ value & AOR $(95 \% \mathrm{Cl})$ & $p$ value \\
\hline \multicolumn{6}{|l|}{ Age (years) } \\
\hline$\leq 5$ & $55 / 67(82.1)$ & 1 & & 1 & \\
\hline 5 to $<10$ & $59 / 105(56.2)$ & $0.28(0.13-0.58)$ & $<0.01$ & $0.28(0.13-0.6)$ & $<0.01$ \\
\hline 10 to $<18$ & $67 / 119(56.3)$ & $0.28(0.14-0.6)$ & $<0.01$ & $0.29(0.14-0.6)$ & $<0.01$ \\
\hline 18 to $<40$ & 72/121 (59.5) & $0.32(0.16-0.66)$ & $<0.01$ & $0.32(0.16-0.7)$ & $<0.01$ \\
\hline 40 to $<90$ & 49/81 (60.5) & $0.33(0.16-0.7)$ & $<0.01$ & $0.33(0.15-0.7)$ & $<0.01$ \\
\hline \multicolumn{6}{|l|}{ IRS } \\
\hline No & & 1 & & & \\
\hline Yes & & $0.52(0.3-1.03)$ & 0.06 & $0.50(0.3-1.0)$ & 0.05 \\
\hline \multicolumn{6}{|l|}{ LLIN at night } \\
\hline No & & 1 & & & \\
\hline Yes & & $0.89(0.5-1.6)$ & 0.69 & & \\
\hline \multicolumn{6}{|l|}{ Gender } \\
\hline Male & & 1 & & & \\
\hline Female & & $0.89(0.6-1.3)$ & 0.53 & & \\
\hline
\end{tabular}

estimated by HS-RDT was similar, even slightly higher, to that determined by PCR, a result partly due to the high proportion of false positives, i.e., samples positive by HSRDT and negative by PCR. The latter could be explained by residual circulating malaria antigen (HRP2) lasting up to 28 days after infection clearance [28, 30], from either anti-malarial treatment [31] or by the immune system [32]. However, some individuals may have been infected with low parasite density fluctuating below the detection threshold of PCR [32-34]. This could be investigated using a larger volume of blood for the molecular analysis [19]. In these individuals with low-density infections, parasite density may increase and are detectable by RDTs or microscopy [35].

Most discrepant results were represented by false negatives, i.e., negative by HS-RDT and positive by PCR, possibly due to the lower detection threshold of PCR compared to HS-RDT [23]. False negative individuals may harbour low-density infections but they may still be infectious to the vector or may progress towards clinical disease [33, 35]. In addition, a false negative result could be due to the deletion of the HRP2 gene [36], HRP2 antigens [11] although this maybe unlikely in this setting. As $P$. falciparum density was not measured, it is not possible to determine whether false negative samples had parasite densities below the HS-RDT detection threshold. Such limitation also prevents to establish whether the differential performance of the HS-RDT by age group and prevalence could be explained by variations in parasite densities. Indeed, HS-RDT's sensitivity and specificity varied by malaria prevalence, with the highest sensitivity and the lowest specificity in high transmission villages. This may be due to the higher probability of being infected (and re-infected) at any given moment (high sensitivity), possibly with higher parasite densities in individuals repeatedly infected, and by the detection of circulating $P$. falciparum HRP2 antigens from past infections (low specificity). Where malaria prevalence was very low, despite missing more than half of infected individuals, HS-RDT would identify those with circulating parasite antigens who had been infected about 1 month earlier $[37,38]$, indicating areas of ongoing transmission.

In this context, treating HS-RDT positive individuals, either with a detectable infection by PCR or with circulating HRP2 from a previous infection, could further decrease malaria prevalence, when using a treatment with a long post-treatment prophylactic period such as dihydroartemisinin-piperaquine (DHA-PQ) [39]. When malaria transmission significantly decreases, it becomes heterogenous and focal, with higher transmission in a few compounds or households and lower transmission in surrounding households [40]. In this context, infected individuals or with evidence of a previous infection may indicate areas of ongoing transmission. This is plausible in The Gambia where households with a malaria-infected individual at the beginning of the transmission season were more likely to have one household member acquire clinical malaria [6].

Trials evaluating the effect of multiple MTAT using conventional RDTs found temporary reductions of malaria prevalence in communities in Burkina Faso and Zambia [14, 41], and in schools in Kenya [16]. Such transient effect is probably due to the inability of conventional RDTs to identify and treat individuals with 

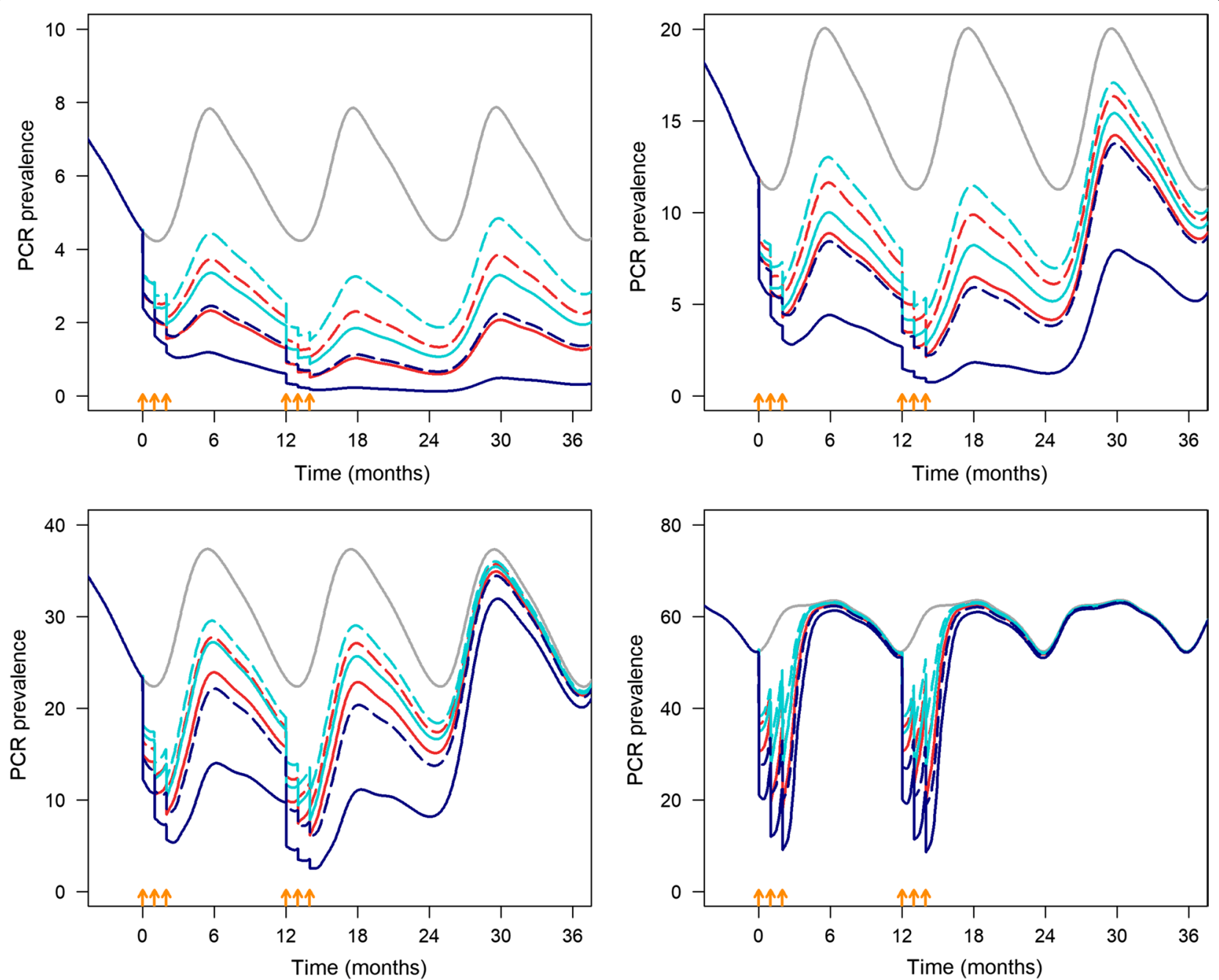

Fig. 6 Mathematical model simulation of the predicted impact of the interventions (MTAT with HS-RDT, MTAT with conventional RDT, and MDA) implemented for three monthly rounds for 2 consecutive years on PCR prevalence. The intervention is implemented in a seasonal transmission setting with four levels of transmission intensity. The orange arrows indicate the timing of each MTAT/MDA round. Solid grey line: no MTAT, dashed light blue line: co-RDT MTAT, 65\% coverage, solid light blue line: co-RDT MTAT, 85\% coverage, dashed red line: HS-RDT MTAT, 65\% coverage, solid red line: HS-RDT MTAT, 85\% coverage, dashed dark blue line: MDA, 65\% coverage, dashed solid dark blue line: MDA, 85\% coverage

low-density infections. In addition, according to a published mathematical model, MTAT with sensitive tests would increase the probability of local malaria elimination only in very low transmission settings [42]. The mathematical model presented in this paper compared MDA and MTAT with either conventional RDT or HSRDT. In all transmission scenarios, MDA would be more efficacious than MTAT in reducing malaria prevalence, although such effect would be transient in areas of intense transmission.

Nevertheless, at very low or low transmission intensities, MTAT with HS-RDT at $85 \%$ coverage would have a similar effect than MDA at $65 \%$ coverage and reduce malaria prevalence by $80 \%$, from 5 to $1 \%$ for about
18 months after the last MTAT round. At lower coverage, MTAT with HS-RDT would have a much lower effect, like that obtained by MTAT with conventional RDT at $85 \%$ coverage. The success of MDA depends on high coverage which in turn is heavily influenced by its acceptability by the local populations, which can change and decrease over time. MTAT may be more acceptable to the local population as it identifies and treats infected individuals [43, 44]. An additional advantage would be to reduce the risk of treatmentrelated adverse events as only HS-RDT positive individuals, in this context a small percentage of the whole population, would be treated. Moreover, MTAT with a HS-RDT may be an alternative to MDA is areas of 
multi-drug resistance [45] as it would reduce the drug pressure and thus the selection of resistant parasites.

The timing of MTAT implementation may also be important; in a highly seasonal setting, the three rounds should be implemented at the end the dry season, before the malaria seasonal transmission starts. This may improve the HS-RDT performance as individuals infected during the previous transmission season would have cleared any residual parasite-circulating antigen, thus reducing the percentage of false positives.

The mathematical model used to estimate the effect on MTAT with HS-RDT is based on several assumptions. These predictions would need to be empirically confirmed by field studies, e.g., cluster randomized trials, which may compare MDA against MTAT or, depending on the malaria prevalence, the two interventions implemented sequentially, i.e., MDA to reduce malaria prevalence to levels at which MTAT could have an additional effect.

\section{Conclusions}

HS-RDTs were developed with the aim of reducing the human reservoir of infection as they would identify asymptomatic, malaria-infected individuals [46] to be treated with an artemisinin-based combination treatment. Despite the sub-optimal sensitivity and specificity, HS-RDT could be used to identify groups of individuals at higher risk of infection. The mathematical model predicts that in low to very low transmission areas, MTAT with HS-RDT would substantially decrease malaria prevalence. Such prediction would need to be confirmed by cluster-randomized trials.

\section{Supplementary information}

Supplementary information accompanies this paper at https://doi. org/10.1186/s12936-019-2929-1.

Additional file 1: Table S1. Comparison of HS-RDT sensitivity and specificity by intensity of transmission.

Additional file 2: Table S2. Risk of being false negative: PCR positive and HS-RDT negative by malaria prevalence.

Additional file 3: Table S3. Risk of false positive: PCR negative and HSRDT positive by transmission intensity.

Additional file 4: Table S4. Percentage reduction in clinical malaria cases over 2 years after simulated MTAT and MDA scenarios shown in Fig. 6.

\section{Abbreviations}

DHA-PQ: dihydroartemisinin-piperaquine; DNA: deoxyribonucleic acid; ELISA: enzyme-linked immunosorbent assay; HDSS: health and demographic surveillance system; HS-RDT: highly sensitive rapid diagnostic test; HRP2: histidine rich protein 2; IRS: indoor residual spraying; LLIN: long-lasting insecticide net; REDCap: research electronic data capture; MDA: mass drug administration; MTAT: mass testing and treatment; PCR: polymerase chain reaction; QGIS: quantum geographic information system; (q)RT-PCR: quantitative reversetranscription polymerase chain reaction; varATS: var gene acidic terminal sequence.

\section{Acknowledgements}

We thank the residents who participated in this study. This study has received support from Mr. Ballah Kandeh, the director of the National Malaria Control Programme, and his staff. We thank the field staff led by Mr. Kebba Naban and all the staff of Malaria Diagnostics Entomology and Epidemiology (MDEE) platform at the MRC Unit Gambia at LSHTM. We thank Mr. Archibald Worwui and Ms. Miriam Wathuo for their statistical support on this article.

\section{Authors' contributions}

JM implemented the study, performed statistical analyses, interpreted the data, wrote the draft and final version of this article. HS, performed the statistical analyses and mathematical modelling, interpreted the data, reviewed draft and final article. JB performed the statistics analyses, interpreted the data, reviewed the draft article and approved the final article. BK provided support in data collection and obtaining approvals. He reviewed and approved the final version of the article. BS provided oversight of the data management, participated in writing the article and approved the final version of the article. FS performed the molecular analyses, interpreted the laboratory data, participated in writing the article and approved the final version. CW performed the mathematical modelling with $\mathrm{HS}$, reviewed the draft manuscripts and approved the final version. DN supervised the molecular analyses, interpretation of the laboratory results, reviewed and approved the final version of the article. TB interpreted the data, participated in writing article and approved the final version of the article. JP and CD interpreted the data, reviewed the draft and final version of the manuscripts. JA participated in design of the study, collection of data, interpretation of results, reviewed the draft and approved final version of the article. UD conceptualized the study, received the grant, designed the study, interpreted the data and reviewed all draft versions of the article and approved the final version. All authors read and approved the final manuscript.

\section{Funding}

This study was funded by DFID/NIHR/MRC/Wellcome Trust global health trials scheme. Grant Number MC_EX_MR_R006075_1.

\section{Availability of data and materials}

The participant data has been collected following provision of informed consent under the prerequisite of strict participant confidentiality (SCC reference 1318). Access to participant data can be accessed following review of the request by the Gambia Government/Medical Research Council Joint Ethics Committee to protect the rights and interests of the study participants. The review process and release of data will be facilitated by Medical Research Council and access will not be unduly restricted. The MRC unit The Gambia at LSHTM is committed to data sharing and open access via its respective policies. Contact information for the Gambia Government/MRC Joint Ethics Committee: Ms Naffie Jobe, Secretary Gambia Government/MRCG at LSHTM Joint Ethics Committee, njobe@mrc.gm.

\section{Ethics approvals and consent to participate}

Ethical approval was obtained from the Gambia Government/Medical Research Council Joint Ethics Committee. Individual written consent was obtained for adults who also provided consent for children less than 18 years of age. Children between 12 and 17 years provided assent.

\section{Consent for publication}

The authors have reviewed the article and provide consent for the publication of these data.

\section{Competing interests}

The authors declare that they have no competing interests.

\section{Author details}

${ }^{1}$ Medical Research Council Unit The Gambia at London School of Hygiene and Tropical Medicine, P.O. Box 273, Banjul, The Gambia. ${ }^{2}$ Faculty of Medicine and Health Sciences, University of Antwerp, Antwerp, Belgium. ${ }^{3}$ MRC Centre for Global Disease Analysis, Department of Infectious Disease Epidemiology, Imperial College London, London, Norfolk Place, London W2 1P, UK. ${ }^{4}$ PATH, 2201 Westlake Avenue, Seattle, USA. ${ }^{5}$ MRC Tropical Epidemiology Group, London School of Hygiene and Tropical Medicine, London, UK. ${ }^{6}$ National Malaria Control Programme, Banjul, Gambia. ${ }^{7}$ Department of Immunology and Infection, Faculty of Infectious Diseases and Tropical Medicine, London School 
of Hygiene and Tropical Medicine, London, UK. ${ }^{8}$ Radboud Institute for Health Sciences, Radboud University Medical Center, Nijmegen, The Netherlands.

Received: 30 March 2019 Accepted: 22 August 2019 Published online: 27 August 2019

\section{References}

1. WHO. World malaria report. Geneva: World Health Organization; 2018.

2. Ministry of Health and Social Welfare, Republic of The Gambia. Malaria Indicator Survey 2017. 2018.

3. Ceesay SJ, Casals-Pascual C, Nwakanma DC, Walther M, Gomez-Escobar $\mathrm{N}$, Fulford AJ, et al. Continued decline of malaria in The Gambia with implications for elimination. PLoS ONE. 2010;5:e12242.

4. Okell LC, Bousema T, Griffin JT, Ouedraogo AL, Ghani AC, Drakeley CJ. Factors determining the occurrence of submicroscopic malaria infections and their relevance for control. Nat Commun. 2012;3:1237.

5. Okell LC, Ghani AC, Lyons E, Drakeley CJ. Submicroscopic infection in Plasmodium falciparum-endemic populations: a systematic review and meta-analysis. J Infect Dis. 2009;200:1509-17.

6. Mwesigwa J, Achan J, Di Tanna GL, Affara M, Jawara M, Worwui A, et al. Residual malaria transmission dynamics varies across The Gambia despite high coverage of control interventions. PLOS ONE. 2017;12:e0187059.

7. Lindblade KA, Steinhardt L, Samuels A, Kachur SP, Slutsker L. The silent threat: asymptomatic parasitemia and malaria transmission. Expert Rev Anti Infect Ther. 2013;11:623-39.

8. WHO. The role of mass drug administration, mass screening and testing, and focal screening and treatment for malaria. Geneva: World Health Organization; 2015 .

9. WHO. Technical consultation on research requirements to support policy recommendations on highly sensitive point-of care diagnostics for $P$. falciparum malaria. Geneva: World Health Organization; 2018. p. 1-34.

10. Laban NM, Kobayashi T, Hamapumbu H, Sullivan D, Mharakurwa S, Thuma PE, et al. Comparison of a PfHRP2-based rapid diagnostic test and PCR for malaria in a low prevalence setting in rural southern Zambia: implications for elimination. Malar J. 2015;14:25

11. Das S, Peck RB, Barney R, Jang IK, Kahn M, Zhu M, et al. Performance of an ultra-sensitive Plasmodium falciparum HRP2-based rapid diagnostic test with recombinant HRP2, culture parasites, and archived whole blood samples. Malar J. 2018;17:118.

12. Bousema T, Okell L, Felger I, Drakeley C. Asymptomatic malaria infections: detectability, transmissibility and public health relevance. Nat Rev Microbiol. 2014;12:833-40.

13. Wu L, van den Hoogen LL, Slater H, Walker PG, Ghani AC, Drakeley CJ, et al. Comparison of diagnostics for the detection of asymptomatic Plasmodium falciparum infections to inform control and elimination strategies. Nature. 2015;528:S86-93.

14. Tiono AB, Ouedraogo A, Ogutu B, Diarra A, Coulibaly S, Gansane A, et al. A controlled, parallel, cluster-randomized trial of community-wide screening and treatment of asymptomatic carriers of Plasmodium falciparum in Burkina Faso. Malar J. 2013;12:79.

15. Scott CA, Yeshiwondim AK, Serda B, Guinovart C, Tesfay BH, Agmas A, et al. Mass testing and treatment for malaria in low transmission areas in Amhara Region, Ethiopia. Malar J. 2016;15:305.

16. von Seidlein $\mathrm{L}$. The failure of screening and treating as a malaria elimination strategy. PLoS Med. 2014;11:e1001595.

17. Halliday KE, Okello G, Turner EL, Njagi K, McHaro C, Kengo J, et al. Impact of intermittent screening and treatment for malaria among school children in Kenya: a cluster randomised trial. PLoS Med. 2014;11:e1001594.

18. Landier J, Haohankhunnatham W, Das S, Konghahong K, Christensen P, Raksuansak J, et al. Operational performance of a Plasmodium falciparum ultrasensitive rapid diagnostic test for detection of asymptomatic infections in Eastern Myanmar. J Clin Microbiol. 2018;56:e00565-18.

19. Hofmann NE, Gruenberg M, Nate E, Ura A, Rodriguez-Rodriguez D, Salib $M$, et al. Assessment of ultra-sensitive malaria diagnosis versus standard molecular diagnostics for malaria elimination: an in-depth molecular community cross-sectional study. Lancet Infect Dis. 2018;18:1108-16.

20. malERA Consultative Group on Diagnoses and Diagnostics. A research agenda for malaria eradication: diagnoses and diagnostics. PLoS Med. 2011;8:e1000396.
21. Imwong M, Hanchana S, Malleret B, Rénia L, Day NP, Dondorp A, et al. High-throughput ultrasensitive molecular techniques for quantifying low-density malaria parasitemias. J Clin Microbiol. 2014;52:3303-9.

22. Das S, Jang IK, Barney B, Peck R, Rek JC, Arinaitwe E, et al. Performance of a high-sensitivity rapid diagnostic test for Plasmodium falciparum malaria in asymptomatic individuals from Uganda and Myanmar and naive human challenge infections. Am J Trop Med Hyg. 2017;97:1540-50.

23. Hofmann N, Mwingira F, Shekalaghe S, Robinson LJ, Mueller I, Felger I. Ultra-sensitive detection of Plasmodium falciparum by amplification of multi-copy subtelomeric targets. PLoS Med. 2015;12:e1001788.

24. WHO. Meeting report of the WHO evidence review group on low-density malaria infection. Geneva: World Health Organization; 2017. p. 1-30.

25. Griffin JT, Bhatt S, Sinka ME, Gething PW, Lynch M, Patouillard E, et al. Potential for reduction of burden and local elimination of malaria by reducing Plasmodium falciparum malaria transmission: a mathematical modelling study. Lancet Infectious Diseases. 2016;16:465-72.

26. Griffin JT, Ferguson NM, Ghani AC. Estimates of the changing age-burden of Plasmodium falciparum malaria disease in sub-Saharan Africa. Nat Commun. 2014;5:3136.

27. Dalrymple U, Arambepola R, Gething PW, Cameron E. How long do rapid diagnostic tests remain positive after anti-malarial treatment? Malar J. 2018;17:228.

28. Iqbal J, Siddique A, Jameel M, Hira PR. Persistent histidine-rich protein 2, parasite lactate dehydrogenase, and panmalarial antigen reactivity after clearance of Plasmodium falciparum monoinfection. J Clin Microbiol. 2004;42:4237-41.

29. Girma S, Cheaveau J, Mohon AN, Marasinghe D, Legese R, Balasingam N, et al. Prevalence and epidemiological characteristics of asymptomatic malaria based on ultrasensitive diagnostics: a cross-sectional study. Clin Infect Dis. 2018. https://doi.org/10.1093/cid/ciy1005 (Epub ahead of print).

30. Nyunt MH, Kyaw MP, Win KK, Myint KM, Nyunt KM. Field evaluation of HRP2 and pan pLDH-based immunochromatographic assay in therapeutic monitoring of uncomplicated falciparum malaria in Myanmar. Malar J. 2013;12:123.

31. Tjitra E, Suprianto S, McBroom J, Currie BJ, Anstey NM. Persistent ICT malaria P.f/P.v panmalarial and HRP2 antigen reactivity after treatment of Plasmodium falciparum malaria is associated with gametocytemia and results in false-positive diagnoses of Plasmodium vivax in convalescence. J Clin Microbiol. 2001;39:1025-31.

32. Felger I, Maire M, Bretscher MT, Falk N, Tiaden A, Sama W, et al. The dynamics of natural Plasmodium falciparum infections. PLOS ONE. 2012;7:e45542.

33. Slater HC, Ross A, Felger I, Hofmann NE, Robinson L, Cook J, et al. The temporal dynamics and infectiousness of subpatent Plasmodium falciparum infections in relation to parasite density. Nat Commun. 2019;10:1433.

34. Farnert A, Snounou G, Rooth I, Bjorkman A. Daily dynamics of Plasmodium falciparum subpopulations in asymptomatic children in a holoendemic area. Am J Trop Med Hyg. 1997;56:538-47.

35. Nguyen T-N, von Seidlein L, Nguyen T-V, Truong P-N, Hung SD, Pham H-T, et al. The persistence and oscillations of submicroscopic Plasmodium falciparum and Plasmodium vivax infections over time in Vietnam: an open cohort study. Lancet Infect Dis. 2018;18:565-72.

36. Amoah LE, Abankwa J, Oppong A. Plasmodium falciparum histidine rich protein-2 diversity and the implications for PfHRP 2: based malaria rapid diagnostic tests in Ghana. Malar J. 2016;15:101.

37. Stresman GH, Kamanga A, Moono P, Hamapumbu H, Mharakurwa S, Kobayashi T, et al. A method of active case detection to target reservoirs of asymptomatic malaria and gametocyte carriers in a rural area in Southern Province, Zambia. Malar J. 2010;9:265.

38. Takem EN, Affara M, Amambua-Ngwa A, Okebe J, Ceesay SJ, Jawara M, et al. Detecting foci of malaria transmission with school surveys: a pilot study in The Gambia. PLoS ONE. 2013:8:e67108.

39. Gutman J, Kovacs S, Dorsey G, Stergachis A, ter Kuile FO. Safety, tolerability, and efficacy of repeated doses of dihydroartemisinin-piperaquine for prevention and treatment of malaria: a systematic review and metaanalysis. Lancet Infect Dis. 2017;7:184-93.

40. Bejon P, Williams TN, Liljander A, Noor AM, Wambua J, Ogada E, et al. Stable and unstable malaria hotspots in longitudinal cohort studies in Kenya. PLoS Med. 2010;7:e1000304. 
41. Larsen DA, Bennett A, Silumbe K, Hamainza B, Yukich JO, Keating J, et al. Population-wide malaria testing and treatment with rapid diagnostic tests and artemether-lumefantrine in southern Zambia: a community randomized step-wedge control trial design. Am J Trop Med Hyg. 2015;92:913-21.

42. Slater HC, Ross A, Ouedraogo AL, White LJ, Nguon C, Walker PG, et al. Assessing the impact of next-generation rapid diagnostic tests on Plasmodium falciparum malaria elimination strategies. Nature. 2015:528:S94-101.

43. Dierickx S, Gryseels C, Mwesigwa J, O'Neill S, Bannister-Tyrell M, Ronse M, et al. Factors associated with non-participation and non-adherence in directly observed mass drug administration for malaria in The Gambia. PLOS ONE. 2016;11:e0148627.
44. Newby G, Hwang J, Koita K, Chen I, Greenwood B, von Seidlein L, et al. Review of mass drug administration for malaria and its operational challenges. Am J Trop Med Hyg. 2015;93:125-34.

45. Zuber JA, Takala-Harrison S. Multidrug-resistant malaria and the impact of mass drug administration. Infect Drug Resist. 2018;11:299-306.

46. Abbott Alere ${ }^{\mathrm{TM}}$ Malaria Ag P.F Ultra sensitive. Abbott. 2019.

\section{Publisher's Note}

Springer Nature remains neutral with regard to jurisdictional claims in published maps and institutional affiliations.
Ready to submit your research? Choose BMC and benefit from:

- fast, convenient online submission

- thorough peer review by experienced researchers in your field

- rapid publication on acceptance

- support for research data, including large and complex data types

- gold Open Access which fosters wider collaboration and increased citations

- maximum visibility for your research: over $100 \mathrm{M}$ website views per year

At BMC, research is always in progress.

Learn more biomedcentral.com/submissions 